Algebraic $\&$ Geometric Topology

Volume 2 (2002) 311-336

Published: 10 May 2002

ATG

\title{
Embedding, compression and fiberwise homotopy theory
}

\author{
JOHN R. KLEIN
}

\begin{abstract}
Given Poincare spaces $M$ and $X$, we study the possibility of compressing embeddings of $M \times I$ in $X \times I$ down to embeddings of $M$ in $X$. This results in a new approach to embedding in the metastable range both in the smooth and Poincaré duality categories.
\end{abstract}

AMS Classification 57P10; 55R99

Keywords Poincaré space, embedding, fiberwise homotopy

\section{Introduction}

Let $M$ and $X$ be compact $n$-manifolds. The word compression of the title refers to a situation in which one is presented with an embedding of $M \times I$ in the interior of $X \times I$ and then tries to decide whether it arises from an embedding of $M$ in $X$, up to isotopy. If so, then the original embedding compresses. One aim of the present paper is to decide when this is possible.

The compression problem is mirrored in the Poincaré duality category. From now on, let $M$ and $X$ be Poincaré duality spaces of dimension $n$. One says that $M$ (Poincaré) embeds in $X$ with complement $C$ if there exists a decomposition $X \simeq M \cup_{\partial M} C$ in which $\partial M \amalg \partial X$ is identified with a Poincaré duality boundary for $C$ (we also assume a compatibility of fundamental classes - see 2.4 below.)

It will be convenient to have separate notation for intervals of different lengths. Let $I=[0,1]$ and $J=[1 / 3,2 / 3]$. For a subspace $S \subset I$ set $M_{S}:=M \times S$. We start with the following data: an embedding of the $(n+1)$-dimensional Poincaré space $M_{J}$ in $X_{I}$ with complement $W$. This gives us a map $\nu: M \rightarrow W$ by taking the composition

$$
M_{1 / 3} \subset \partial M_{J} \rightarrow W .
$$

Let $R(X)$ denote the category of retractive spaces over $X$. An object of $R(X)$ is a space $Y$ equipped with maps $s_{Y}: X \rightarrow Y$ and $r_{Y}: Y \rightarrow X$ (called respectively inclusion and retraction) such that $r_{Y} \circ s_{Y}$ is the identity (objects 
are usually specified without reference to their structure maps). A morphism $Y \rightarrow Z$ is a map of spaces which is compatible with the structure maps. According to Quillen [16], $R(X)$ is a model category in which a weak equivalence is a morphism $Y \rightarrow Z$ which when considered as a map of spaces is a weak homotopy equivalence (for the remaining structure, see 2.1 below). Hence, it makes sense to speak of its homotopy category hoR $(X)$.

The inclusion $X_{0} \subset W$ and the composite $W \rightarrow X_{I} \rightarrow X$ equip the space $W$ with the structure of an object of $R(X)$. Let $M^{+}$denote the object of $R(X)$ given by taking the disjoint union of $M$ with $X$; the inclusion $X \rightarrow M^{+}$is evident and the retraction $M^{+} \rightarrow X$ is defined to be the composite

$$
M \amalg X=M_{1 / 3} \amalg X_{0} \subset M_{J} \amalg X_{0} \rightarrow X_{I} \stackrel{\text { project }}{\longrightarrow} X \text {. }
$$

With respect to these conventions, the map $\nu: M \rightarrow W$ induces a morphism

$$
\nu^{+}: M^{+} \rightarrow W
$$

of $R(X)$. Then $\nu^{+}$determines a fiberwise homotopy class

$$
\left[\nu^{+}\right] \in\left[M^{+}, W\right]_{X} .
$$

Remark 1.1 This will be the primary obstruction to compression. Informally, it should be thought of as measuring the self-linking of $M$ in $X_{I}$. Several authors have studied non-fiberwise versions of this construction (see Hirsch [7], Levitt [14] and Williams [22]).

Following Goodwillie [4], the homotopy codimension of $M$ is $\geq q$, if

- $M$ is homotopy equivalent to a CW complex of $\operatorname{dim} \leq n-q$, and

- the inclusion $\partial M \rightarrow M$ is $(q-1)$-connected.

In what follows, we write $\operatorname{codim} M \geq q$. By a result of Wall [19], the first condition is a consequence of the second whenever $q \geq 3$.

Examples 1.2 (1) If $M$ is regular neighborhood of $p$-dimensional complex in an $n$-dimensional manifold, then $\operatorname{codim} M \geq n-p$.

(2) Let $V^{p}$ be a closed Poincaré space of dimension $p$ equipped with an $(n-p-1)$-spherical fibration $\xi: S(\xi) \rightarrow V$. Let $D(\xi)$ be the mapping cylinder of $\xi$. Then $(D(\xi), S(\xi))$ is a Poincaré pair of dimension $n$ with $\operatorname{codim} D(\xi) \geq$ $n-p$.

We now state the main result. 
Theorem A Assume codim $M \geq n-p \geq 3$ and $3 p+4 \leq 2 n$. Then there exists an embedding of $M$ in $X$ which induces the given embedding of $M_{J}$ in $X_{I}$ (up to "concordance") if and only if $\left[\nu^{+}\right] \in\left[M^{+}, W\right]_{X}$ is trivial.

We remark that this is valid in both the smooth and Poincare cases (the smooth case follows by application of the surgery machine - see below). In the special case $X=D^{n}$ is a disk, Theorem A reduces to a non-fiberwise result which is implicit in the work of Williams [23]. In fact, our proof of Theorem A is a fiberwisation of one of Williams' arguments.

With respect to the numerical assumptions of Theorem A, we have

Addendum 1.3 The map of fiberwise homotopy classes

$$
\Sigma_{X}:\left[M^{+}, W\right]_{X} \rightarrow\left[\Sigma_{X} M^{+}, \Sigma_{X} W\right]_{X}
$$

is an isomorphism, where $\Sigma_{X}$ denotes fiberwise suspension. Consequently, the obstruction to compression $\left[\nu^{+}\right]$is stable.

This is proved in $\S 7$ using the Freudenthal suspension theorem for ho $R(X)$ (cf. 2.3 below).

\section{$1.1 \quad$ Unstable fiberwise normal invariants}

Let $M$ and $X$ be $n$-dimensional Poincaré spaces, and let $f: M \rightarrow X$ be a map. These data define an object

$$
M / / \partial M \in R(X)
$$

whose underlying space is $X \cup_{f \mid \partial M} M$ (note: collapsing $X$ to a point gives the quotient $M / \partial M)$. Similarly, we have $X / / \partial X \in R(X)$ which turns out to be the double $X \cup_{\partial X} X$ (which gives $X^{+}$if $\partial X$ is empty.)

If $f: M \rightarrow X$ is the underlying map of an embedding of $M$ in $X$ with complement $C$, then there is an associated fiberwise homotopy class

$$
\alpha_{f} \in[X / / \partial X, M / / \partial M]_{X}
$$

defined by taking

$$
X \cup_{\partial X} X \stackrel{\simeq}{\longleftarrow} X \cup_{\partial X}\left(C \cup_{\partial M} M\right) \longrightarrow X \cup_{X}\left(X \cup_{\partial M} M\right)=M / / \partial M .
$$

This is the fiberwise (Thom-Pontryagin) collapse of the embedding.

By analogy with Smale-Hirsch theory, a map $f: M \rightarrow X$ is said to (Poincaré) immerse if there exists an integer $j \geq 0$ such that $f \times \mathrm{id}: M \times D^{j} \rightarrow X \times D^{j}$ is the underlying map of some embedding. 
Remark 1.4 A fact we won't need, but which is nevertheless true, is that $f$ Poincaré immerses if and only if there is a stable fiber homotopy equivalence $f^{*} \nu_{X} \simeq \nu_{M}$, where $\nu_{X}$ and $\nu_{M}$ denote the Spivak normal fibrations of $X$ and $M$ respectively. For a proof of this, see [12].

Taking the fiberwise collapse of the embedding $M \times D^{j} \rightarrow X \times D^{j}$ enables us to associate a fiberwise stable homotopy class

$$
\alpha_{f}^{\mathrm{st}} \in\{X / / \partial X, M / / \partial M\}_{X}
$$

called the fiberwise (stable) normal invariant of the immersion (this is independent of the choice of embedding.)

Obviously, a necessary obstruction to compressing the given embedding to an embedding of $M$ in $X$ is that $\alpha_{f}^{\text {st }}$ should desuspend to an element $\alpha_{f} \in$ $[X / / \partial X, M / / \partial M]_{X}$. Call any such desuspension a fiberwise unstable normal invariant of the immersion.

Theorem B Assume $f: M \rightarrow X$ immerses. Again, suppose that codim $M \geq$ $n-p \geq 3$ and $3 p+4 \leq 2 n$. Then $f$ embeds (inducing the given immersion) if and only if there exists a fiberwise unstable normal invariant $\alpha_{f}$. Moreover, the embedding can be chosen so that its collapse induces $\alpha_{f}$.

In the case $\partial X=\emptyset$, Richter has also proved Theorem B using fiberwise Hopf invariants and fiberwise $S$-duality. By contrast, we will deduce Theorem B from Theorem A (in fact, the theorems are equivalent).

A consequence of the above is a Whitney embedding theorem for immersions in the Poincaré duality category:

Corollary C Assume $f: M^{p} \rightarrow X^{n}$ immerses, where codim $M \geq n-p \geq 3$ and $2 p+1 \leq n$. Then $f$ embeds (inducing the given immersion up to concordance).

This follows because the fiberwise stable normal invariant destabilizes by 2.3 .

\subsection{A Levine style embedding theorem}

When $X$ is 'highly' connected, Theorem B simplifies to a non-fiberwise statement. Here is its formulation: given an immersion of $f: M \rightarrow X$ as above, there is an associated stable (Thom-Pontryagin) collapse

$$
\beta^{\text {st }} \in\{X / \partial X, M / \partial M\} .
$$


Any homotopy class

$$
\beta \in[X / \partial X, M / \partial M]
$$

which suspends to $\beta^{\text {st }}$ is called an unstable normal invariant.

Theorem D Assume codim $M \geq n-p \geq 3, X$ is $[p / 2]$-connected and $3 p+4 \leq$ $2 n$. Then there exists an embedding inducing the given immersion of $M$ in $X$ if and only if there exists an unstable normal invariant $\beta$. Moreover, the embedding can be chosen so that its collapse coincides with $\beta$.

For example, if we take $X=D^{n}$ then we recover the Williams-Richter embedding theorem [23], [17]. Levine's embedding theorem [13, Thm. 4] amounts to the case when $X$ is a smooth $n$-manifold and $M=D(\xi)$ is the unit disk bundle of a vector bundle over a smooth $p$-manifold $V$.

\subsection{Embedding spheres in the middle dimension}

In applications to surgery on Poincaré spaces, one of the main issues is whether or not homotopy classes in the middle dimension are represented by 'framed' embedded spheres.

Let $X^{n}$ be a Poincaré space, and suppose that $n=2 p$. Set

$$
P:=S^{p} \times D^{p},
$$

and suppose that $f: P \rightarrow X$ is a map which immerses. Let $\widetilde{X}$ be the universal cover of $X$, and let $\pi$ be the group of deck transformations. A map $Y \rightarrow X$ then induces a $\pi$-covering of $Y$ which we denote by $\widetilde{Y}$. Note that $\widetilde{X} / \partial \widetilde{X}$ is a based $\pi$-space, which is free in the based sense.

The immersion $f$ gives rise to an equivariant stable homotopy class

$$
\widetilde{\beta}^{\text {st }} \in\{\widetilde{X} / \partial \widetilde{X}, \widetilde{P} / \partial \widetilde{P}\}^{\pi},
$$

called the equivariant stable collapse. This is constructed as follows: choose a representative embedding for $f \times \operatorname{id}_{D^{j}}: P \times D^{j} \rightarrow X \times D^{j}$. The diagram for this embedding can then be pulled-back along $\widetilde{X}$. The Thom-Pontryagin collapse of the resulting diagram of $\pi$-spaces then yields $\widetilde{\beta}^{\text {st }}$.

Theorem E Assume $p>2$. An immersion $f: P \rightarrow X$ is represented by an embedding if and only if the equivariant stable collapse desuspends to an element $\widetilde{\beta} \in[\widetilde{X} / \partial \widetilde{X}, \widetilde{P} / \partial \widetilde{P}]^{\pi}$. Furthermore, the embedding can be chosen so that its equivariant collapse is $\widetilde{\beta}$. 


\subsection{Embedded thickenings}

Up until now, we have discussed embedding theorems between Poincaré spaces having the same dimension. In a previous paper [11], we studied the following related problem: suppose that $K$ is the homotopy type of a finite complex, $X^{n}$ is a Poincaré space, and $f: K \rightarrow X$ is a map. Does there exist a 'Poincaré boundary' for $K$, say $A \rightarrow K$, such that $f: K \rightarrow X$ embeds? (More precisely, we should really replace $K$ by the mapping cylinder of the map $A \rightarrow K$ to get a Poincaré pair.) Additionally, one assumes a codimensional restriction: $k \leq n-3$, where $k$ is the homotopy dimension of $K$ (an integer such that $K$ is homotopy equivalent to a $\mathrm{CW}$ complex of that dimension).

This is the notion of Poincaré embedding in which the 'normal data' are not $a$ priori chosen. In [11] we termed this notion a $P D$ embedding. In this paper, we will call it an embedded thickening, since the choice of Poincaré boundary is a 'Poincaré thickening' of $K$.

An important special case of this concept is when $K$ itself is a closed Poincaré space. In this instance, the homotopy fiber of the map $A \rightarrow K$ is a sphere, and one recovers the notion of Poincaré embedding used by Wall [21, Chap. 11].

In [11], we proved that $f: K^{k} \rightarrow X^{n}$ embedded thickens whenever $f$ is $(2 k-$ $n+2)$-connected. It was expected that this is not the sharpest result, for in the smooth case, this result can be improved by one dimension. We show that the result can be improved by one dimension in the range of Theorem A:

Theorem F Assume $f: K \rightarrow X$ is $(2 k-n+1)$-connected, $k \leq n-3$ and $3 k+4 \leq 2 n$. Then there exists an embedded thickening of $f$.

Note that this immediately implies the Poincaré versions of the 'easy' and 'hard' Whitney embedding theorems: let $f: K \rightarrow X$ be a map with $k \leq n-3$.

Corollary G (1) If $2 k+1 \leq n$, then $f$ embedded thickens.

(2) If $2 k \leq n$ and and $f$ is 1-connected, then $f$ embedded thickens with the possible exception of the case $k=3$ and $n=6$.

Remark 1.5 The first part of the corollary settles an issue raised by Levitt [14, p. 402].

Another application yields an extension of [11, Cor. C], which concerns the existence of the unstable homotopy tangent bundle for Poincaré spaces: 
Corollary $\mathbf{H}$ Let $X^{n}$ be a 1-connected closed Poincaré space. Then the diagonal $X \rightarrow X \times X$ has an embedded thickening.

This follows by Theorem $\mathrm{F}$ if $n \geq 4$, and is trivial if $n<4$.

\subsection{Smooth embeddings}

If $M$ and $X$ are compact smooth manifolds, then the Browder-Casson-SullivanWall theorem [21, Chap. 11] shows that all of the above results imply smooth embedding results, (some new, some known). We leave it to the reader to make sense of this translation.

The inequality $3 p+4 \leq 2 n$ can be improved to $3 p+3 \leq 2 n$ in the smooth case: in proving Theorem A we make use of the relative embedding theorem of [10], which is the Poincaré variant of a result of Hodgson [8] with a loss of one dimension. In the smooth case, Hodgson's result may be directly substituted in the appropriate part of the proof of Theorem A to yield the sharper result.

\subsection{History}

The concept of Poincaré embedding surfaced in an attempt to understand smooth embeddings within the framework of surgery theory. The BrowderCasson-Sullivan-Wall theorem asserts that the smooth embedding problem of $M^{n}$ in $X^{n}$ is equivalent to the corresponding Poincaré embedding problem as long as $n \geq 6$ and codim $M \geq 3$. Consequently, the problem of smooth embedding is reduced to homotopy theory.

The inequality $3 p+3 \leq 2 n$ is called the metastable range. Roughly, it is the place where triple point obstructions don't arise for dimensional reasons.

From 1960-1975 there emerged (at least) three different strategies to (smooth) embedding in the metastable range. Firstly, there was the school of Haefliger, which reduced the problem to a question about isovariant maps $M^{\times 2} \rightarrow X^{\times 2}$ (an equivariant map such that the inverse image of the diagonal of $X$ coincides with the diagonal of $M$ ). Secondly, there was the bordism theoretic approach, as seen in the papers of Dax [3] and Hatcher-Quinn [6]. Both of these schools relied heavily on the Whitney trick and/or engulfing methods.

Lastly, there was the surgery school-most notably the works of Browder [1], [2] and Wall [21] — which reduced the problem of smooth embedding to that of Poincaré embedding. This approach began with Levine [13], who, using surgery, 
constructed embeddings from unstable normal invariants when the source $M$ is the total space of a disk bundle over a smooth manifold and the ambient space $X$ is an $n$-sphere, or more generally when $X$ is a sufficiently highly connected manifold. Here, the role of the normal bundle is prominent.

Later, Williams [23], [22], Rigdon-Williams [18] and Richter [17], extended Levine's work to the case when $M$ is a Poincaré space and $X=D^{n}$. The work of Williams et. al. used smooth manifold techniques to deduce results about Poincaré embeddings. Richter gave the first manifold-free proof of Williams' results using homotopy theory.

It was only recently observed [11] that fiberwise homotopy theory technology was to play a role in extending the surgery approach to an arbitrary ambient Poincaré space $X$. This connection was discovered by Shmuel Weinberger and the author (independently). The present work is an attempt to complete the thread begun by the surgery school.

\subsection{Outline}

Section 2 is mostly language; the reader should be familiar with the majority of material in this section. In $\S 3$ we show that the existence of a fiberwise normal invariant is sufficient to give an embedding of $M_{J}$ in $X_{I}$ whose obstruction to compression is trivial, so Theorem A implies the first part of Theorem B. $\S 4$ concerns the proof of Theorems D and E, which are a consequence of Theorem B and Milgram's EHP sequence. In $\S 5$ we prove Theorem A. The main tool in the proof is the relative embedded thickening theorem of [10]. In $\S 6$ we show that the embedding constructed in $\S 3$ has the correct collapse, thereby completing the proof of Theorem B. In $\S 7$ we prove the stability of the obstruction $\left[\nu^{+}\right]$. In $\S 8$ we prove Theorem $\mathrm{F}$.

\subsection{Acknowledgments}

This paper could not have been written were it not for discussions I had with Tom Goodwillie and Bill Richter. The proof of Theorem A was in part motivated by techniques employed by Goodwillie to study the stability map in relative pseudoisotopy theory. As I mentioned above, the first proof of Theorem B is due to Richter. Also, the idea of the proof of 8.2 was aided by interaction with Richter. Thanks to Andrew Ranicki for improvements in the exposition. Lastly, I've benefited from the papers of Bruce Williams. 


\section{Preliminaries}

Our ground category is Top, the category of compactly generated Hausdorff spaces. This comes equipped with the structure of a Quillen model category:

- The weak equivalences are the weak homotopy equivalences (i.e., maps $X \rightarrow Y$ such that the associated realization of its singular map $|S . X| \rightarrow$ $|S . Y|$ is a homotopy equivalence). Weak equivalences are denoted $\stackrel{\sim}{\rightarrow}$.

- The fibrations, denoted $\rightarrow$, are the Serre fibrations.

- The cofibrations, denoted $\longmapsto$, are the 'Serre cofibrations', i.e., inclusion maps given by a sequence of cell attachments (i.e., relative cellular inclusions) or retracts thereof.

Every object is fibrant. The cofibrant objects are the retracts of iterated cell attachments built up from the empty space. Every object $Y$ comes equipped with a functorial cofibrant approximation $Y^{\mathrm{c}} \stackrel{\sim}{\rightarrow} Y$.

A non-empty space is always $(-1)$-connected. A connected space is 0 -connected, and is $r$-connected for some $r>0$ if its homotopy groups vanish up through degree $r$, for any choice of basepoint. A map of non-empty spaces $X \rightarrow Y$ is called $r$-connected if its homotopy fiber with respect to any choice of basepoint in $Y$ is an $(r-1)$-connected space. An $\infty$-connected map is a weak equivalence.

A space is homotopy finite if it is homotopy equivalent to a finite $\mathrm{CW}$ complex.

A commutative square of cofibrant spaces

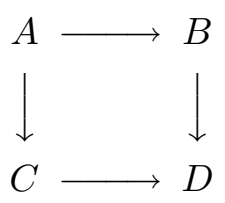

is $r$-cocartesian if the evident map $C_{0} \cup A_{[0,1]} \cup B_{1} \rightarrow D$ (whose source is a double mapping cylinder) is $r$-connected. More generally, a square of not necessarily cofibrant spaces is $r$-cocartesian if it is after applying cofibrant approximation. An $\infty$-cocartesian square is cocartesian. Dually, the square is $r$-cartesian if the map $A \rightarrow \operatorname{holim}(B \rightarrow D \leftarrow C)$ is $r$-connected. An $\infty$-cartesian square is cartesian.

We introduce one last non-standard notation: given a map of spaces $A \rightarrow B$, if no confusion arises we will often let $(\bar{B}, A)$ denote the pair given by the mapping cylinder $B_{0} \cup A_{I}$ with the inclusion of $A_{1}$.

Algebraic $\mathcal{E} \mathcal{G}$ Geometric Topology, Volume 2 (2002) 


\subsection{Fiberwise spaces}

For $X \in$ Top an object, $R(X)$ will denote the category of retractive spaces, as in the introduction (in another notation, not to be used here, $X \backslash \mathbf{T o p} / X$ ). We will assume in what follows that $X$ is a cofibrant object of Top.

According to Quillen [16], $R(X)$ inherits a model category structure arising from the one on Top. Weak equivalences and fibrations are defined using the forgetful functor $R(X) \rightarrow$ Top. Cofibrations are those maps satisfying the left lifting property with respect to the acyclic fibrations (the word 'acyclic' is synonymous with weak equivalence).

Any object $Y \in R(X)$ comes equipped with a functorial cofibrant approximation $Y^{\mathrm{c}} \stackrel{\cong}{\hookrightarrow} Y$ and similarly, a functorial fibrant approximation $Y \stackrel{\sim}{\sim} Y^{\mathrm{f}}$.

Given an object $Y \in R(X)$, define its fiberwise suspension $\Sigma_{X} Y$ to be the object whose underlying space is obtained by collapsing the subspace $X_{I} \subset$ $\Sigma_{X} Y$ to $X$ (via the first factor projection) in the double mapping cylinder $X_{0} \cup Y_{I} \cup X_{1}$. If $Y$ is cofibrant, then so is its fiberwise suspension. We use the notation $\Sigma_{X}^{j} Y$ to denote the $j$-fold iterated application of $\Sigma_{X}$ to $Y$.

The homotopy category of $R(X)$, denoted hoR(X), is the category whose objects are those of $R(X)$ and in which the hom-set from an object $Y$ to an object $Z$ is given by homotopy classes of morphisms $Y^{\mathrm{c}} \rightarrow Z^{\mathrm{f}}$. This is denoted $[Y, Z]_{X}$; it is a pointed set. The corresponding stable hom-set is $\{Y, Z\}_{X}:=\lim _{j}\left[\Sigma_{X}^{j} Y, \Sigma_{X}^{j} Z\right]_{X}$.

Obstruction theory in Top gives rise to an obstruction theory in $R(X)$. Let $Z \in R(X)$ be an object. A commutative diagram

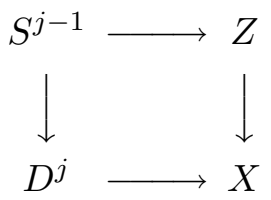

defines another object $Z \cup D_{X}^{j}$, whose underlying space is $Z \cup_{S^{j-1}} D^{j}$. This operation is called attaching a $j$-cell to $Z$.

Definition 2.1 An object $P \in R(X)$ has dimension $\leq s$ if its fibrant approximation admits a factorization $X \longmapsto P^{\prime} \stackrel{\sim}{\rightarrow} P^{\mathrm{f}}$ such that $P^{\prime}$ is obtained from $X$ by attaching cells of dimension $\leq s$.

A morphism $Y \rightarrow Z$ is $r$-connected if it is $r$-connected as a map of spaces. In particular, an object $Y$ is $r$-connected if its structure map $X \rightarrow Y$ is. 
Lemma 2.2 Let $Y \rightarrow Z$ be $r$-connected morphism of $R(X)$ and suppose that $P$ has dimension $\leq r$. Then the induced map of homotopy sets

$$
[P, Y]_{X} \rightarrow[P, Z]_{X}
$$

is surjective. It is also injective if $P$ has dimension $\leq r-1$.

This is essentially $[9,9.2]$.

\subsection{The stable range}

The Freudenthal theorem measures the extent to which fiberwise suspension is an isomorphism on the level of fiberwise homotopy classes.

Lemma 2.3 (James $[9,9.3]$ ). If $Y, Z \in R(X)$ cofibrant objects such that $Z$ $r$-connected and $Y$ has dimension $\leq 2 r+1$, then fiberwise suspension gives a surjection of pointed sets

$$
[Y, Z]_{X} \rightarrow\left[\Sigma_{X} Y, \Sigma_{X} Z\right]_{X}
$$

This surjection is an isomorphism whenever $Y$ has dimension $\leq 2 r$.

\subsection{Poincaré spaces}

In this paper, a Poincaré space $X$ of dimension $n$ is a pair $(X, \partial X)$ such that $X$ and $\partial X$ are cofibrant and homotopy finite, $\partial X \rightarrow X$ is a cofibration, and $X$ satisfies Poincaré duality:

- there exists a local system of abelian groups $\mathcal{L}$ of rank one defined on $X$, and a fundamental class $[X] \in H_{n}(X, \partial X ; \mathcal{L})$ such that the cap product homomorphisms

$$
\cap[X]: H^{*}(X ; M) \rightarrow H_{n-*}(X, \partial X ; \mathcal{L} \otimes M)
$$

and

$$
\cap[\partial X]: H^{*}(\partial X ; N) \rightarrow H_{n-*-1}\left(\partial X ; \mathcal{L}_{\mid \partial X} \otimes N\right)
$$

are isomorphisms, where $[\partial X] \in H_{n-1}\left(\partial X ; \mathcal{L}_{\mid \partial X}\right)$ is the image of $[X]$ under the connecting homomorphism in the homology exact sequence of the pair $(X, \partial X)$, and $M(N)$ is any local system on $X$ (resp. on $\partial X$ ) (compare [11], [20]).

If $(X, \partial X)$ is a pair such that $\partial X \rightarrow X$ is 2-connected, then the first duality isomorphism implies the second one (cf. $[11,2.1])$. In these circumstances, $X$ is $n$-dimensional Poincaré if and only if $X_{I}$ is $(n+1)$-dimensional Poincaré. 


\subsection{Embeddings}

Let $M$ and $X$ a Poincaré spaces of dimension $n$, where $X$ is connected. An embedding of $M$ in $X$ is a commutative cocartesian square of cofibrant homotopy finite spaces

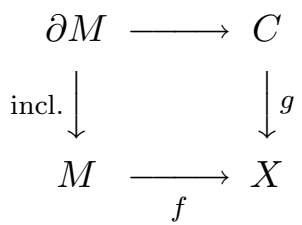

together with a factorization of the inclusion $\partial X \rightarrow C \rightarrow X$, such that $(M, \partial M)$ and $(\bar{C}, \partial M \amalg \partial X)$ satisfy Poincaré duality with respect to the fundamental classes obtained by taking the image of a fundamental class for $X$ under the homomorphisms

$$
H_{n}(X, \partial X ; \mathcal{L}) \rightarrow H_{n}(\bar{X}, C ; \mathcal{L}) \cong H_{n}\left(M, \partial M ; f^{*} \mathcal{L}\right)
$$

and

$$
H_{n}(X, \partial X ; \mathcal{L}) \rightarrow H_{n}(X, M \amalg \partial X ; \mathcal{L}) \cong H_{n}\left(\bar{C}, \partial M \amalg \partial X ; g^{*} \mathcal{L}\right) .
$$

If codim $M \geq 3$ then one only need verify the compatibility of fundamental classes for $M$ (see $[11,2.3])$.

The space $C$ is called the complement, and $f: M \rightarrow X$ is the underlying map of the embedding.

The decompression of an embedding of $M$ in $X$ is the embedding of $M_{I}$ in $X_{I}$ defined by the diagram

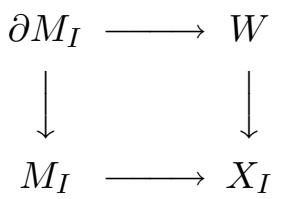

where $W=X_{0} \cup C_{I} \cup X_{1}$ is (unreduced) fiberwise suspension, and the factorization $\partial X_{I} \rightarrow W \rightarrow X_{I}$ is evident.

Two embeddings from $M$ to $X$ with complements $C_{0}$ and $C_{1}$ are elementary concordant if there exists a diagram of pairs

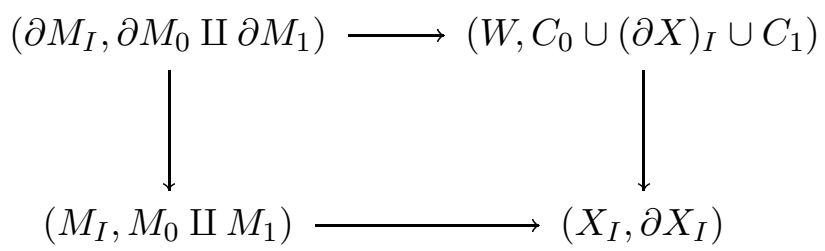

Algebraic 83 Geometric Topology, Volume 2 (2002) 
in which each associated diagram of spaces

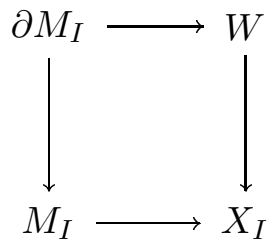

and

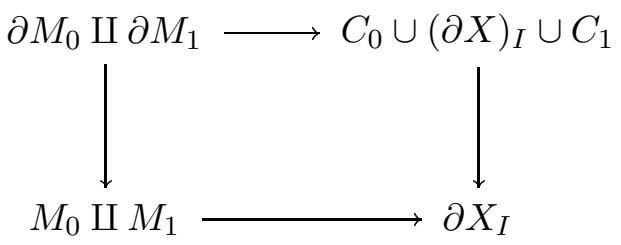

is cocartesian (the latter of these is obtained from the disjoint union of the embedding diagrams using the inclusion $\left.\partial X_{0} \amalg \partial X_{1} \subset \partial X_{I}\right)$. Moreover, the maps $C_{i} \rightarrow W$ are required to be weak equivalences. More generally, concordance is the equivalence relation generated by elementary concordance.

\subsection{Embedded thickenings}

Suppose that $K$ is a cofibrant space which is homotopy equivalent to a finite connected CW complex of dimension $\leq k$. Let $f: K \rightarrow X$ be a map, where $X^{n}$ is a connected Poincaré space of dimension $n$. A cocartesian square

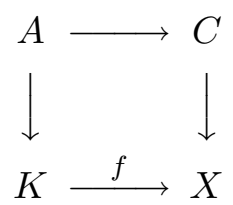

(in which $A$ and $C$ are cofibrant and homotopy finite), together with a factorization $\partial X \rightarrow C \rightarrow X$ is called an embedded thickening of $f$ if

- $(\bar{K}, A)$ gives an $n$-dimensional Poincaré space such that codim $\bar{K} \geq n-k$, and

- Replacing $K$ by $\bar{K}$ in the diagram yields an embedding in the sense of 2.4 .

An embedded thickening is what was called a $P D$ embedding in the terminology of [11]. In order to avoid confusion, we have changed the name to distinguish between the embeddings appearing in this paper (where the boundary data are a priori given) and the ones of [11] (embeddings of complexes in Poincaré spaces).

\section{Proof of Theorem B (first part)}

We show how Theorem A can be used to construct an embedding of $M$ in $X$ from an unstable fiberwise normal invariant. 
Let $\alpha_{f} \in[X / / \partial X, M / / \partial M]_{X}$ be an unstable fiberwise normal invariant associated to an immersion $f: M \rightarrow X$. Based on a construction of Browder [2] we will associate a Poincaré embedding of $M_{J}$ in $X_{I}$.

For this section only, let us agree that $M / / \partial M$ now means the object of $R(X)$ whose underlying space is $X_{0} \cup(\partial M)_{I} \cup M_{1}$ (the formulation provided in the introduction differs from this description by a canonical weak equivalence). Similarly, let $X / / \partial X$ now mean $X_{0} \cup(\partial X)_{I} \cup X_{1}$. Let $h: J \rightarrow I$ be the homeomorphism $t \mapsto 3 t-1$.

Then there is a commutative diagram of spaces

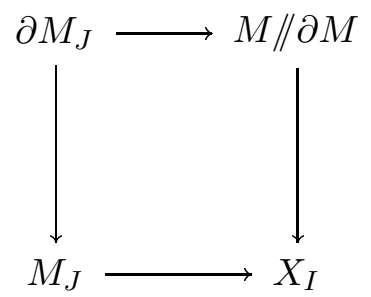

in which the top arrow is defined by

$$
M_{1 / 3} \cup(\partial M)_{J} \cup M_{2 / 3} \stackrel{\operatorname{id} \times h}{\longrightarrow} M_{0} \cup(\partial M)_{I} \cup M_{1} \stackrel{f \cup i d \cup i d}{\longrightarrow} X_{0} \cup(\partial M)_{I} \cup M_{1},
$$

the bottom arrow is $f \times h$, and the vertical arrows are evident. This diagram is cocartesian. In what follows, we must replace $M / / \partial M$ in the diagram with its fibrant approximation $(M / / \partial M)^{\mathrm{f}}$. Assume that this has been done.

The Poincaré boundary for $X_{I}$ is $X / / \partial X$; it factors through $(M / / \partial M)^{\mathrm{f}}$ via a representative for $\alpha_{f}$. This defines the embedding of $M_{J}$ in $X_{I}$. In particular, the complement of this embedding is $(M / / \partial M)^{\mathrm{f}}$.

Applying Theorem A, we see that the given embedding compresses to a embedding of $M$ in $X$ if and only if $\left[\nu^{+}\right] \in\left[M^{+}, M / / \partial M\right]_{X}$ is the trivial element. But by construction, $\left[\nu^{+}\right]$is the fiberwise homotopy class determined by making the composite (fiberwise) map

$$
M_{1 / 3} \rightarrow M_{1 / 3} \cup(\partial M)_{J} \cup M_{2 / 3} \rightarrow X_{0} \cup(\partial M)_{I} \cup M_{1}
$$

"based" (i.e., add on a disjoint copy of $X$ to $M_{1 / 3}$ ). The composite clearly factors through the "basepoint" $X_{0} \subset X_{0} \cup(\partial M)_{I} \cup M_{1}$, so $\left[\nu^{+}\right]$is the trivial element.

It remains to check that the collapse of the embedding of $M$ in $X$ equals $\alpha_{f}$. This is not a formal consequence of Theorem A, but rather, a consequence of the construction of the particular embedding in the proof of Theorem A contained in $\S 5$ below. For this reason, we defer the proof of this until $\S 6$. 


\section{Proof of Theorems D and E}

Proof of Theorem D We first explain the idea of the proof while ignoring technical details. There is a commutative diagram of $R(X)$

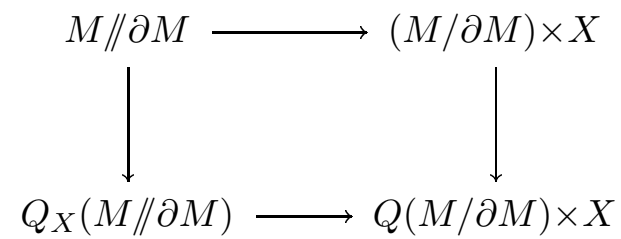

in which

- $(M / \partial M) \times X$ has structure maps given by the second factor projection and the inclusion $* \times X \subset(M / \partial M) \times X$.

- The morphism $M / / \partial M \rightarrow(M / \partial M) \times X$ is given by the quotient map $M / / \partial M \rightarrow M / \partial M$ together with the retraction $M / / \partial M \rightarrow X$.

- $Q_{X}$ means the fiberwise version of stable homotopy, and the bottom map of the diagram is defined in a way similar to the top map.

- The vertical maps are defined by means of the natural transformation from the identity to (fiberwise) stable homotopy.

Ignoring for the moment the issue of homotopy invariance of the terms in the diagram, it will follow by an argument sketched below that the diagram is $n$-cartesian. Assuming this the argument proceeds as follows:

The fiberwise stable homotopy class $\alpha^{\text {st }}$ is represented by a morphism $X / / \partial X \rightarrow$ $Q_{X}(M / / \partial M)$ and the homotopy class $\beta$ is represented by a morphism $X / / \partial X \rightarrow$ $(M / \partial M) \times X$. Up to fiberwise homotopy the maps are compatible with the diagram. By 2.2 applied to the $n$-connected morphism

$$
M / / \partial M \rightarrow \operatorname{holim}\left(Q_{X}(M / / \partial M) \rightarrow Q(M / \partial M) \times X \leftarrow(M / \partial M) \times X\right)
$$

there is an unstable fiberwise normal invariant $\alpha \in[X / / \partial X, M / / \partial M]_{X}$. Theorem $\mathrm{D}$ now follows by application of Theorem B.

We now proceed to establish the degree to which the square is cartesian. First of all, we replace the square by an equivalent one which is homotopy invariant (for the extent to which $Q_{X}$ is a homotopy invariant functor is unclear, even for objects which are fibrant and cofibrant).

Choose a basepoint for $X$. Since $X$ is a connected cofibrant space, there is a homotopy equivalence $X \simeq B G$ where $G$ is the geometric realization of the 
simplicial set given which is the Kan loop group of the total singular complex of $X$. Here, we think of $G$ as a topological group object in Top. In what follows, we will assume $X$ is $B G$.

Let $R^{G}(*)$ denote the category of based $G$-spaces. This admits the structure of a model category in which a morphism $Y \rightarrow Z$ is a weak equivalence if (and only if) it is a weak homotopy equivalence of spaces. Every object is fibrant and the cofibrant objects are the retracts of free based $G$-CW complexes. In fact, the homotopy categories of $R^{G}(*)$ and $R(B G)$ are equivalent (but we will not require this.)

Let $M^{\sim}$ denote the pullback of $M \rightarrow B G \leftarrow E G$. Then $M^{\sim} / \partial M^{\sim}$ is an object of $R^{G}(*)$. We recover $M / / \partial M \in R(B G)$ up to weak equivalence by taking the Borel construction $\left(M^{\sim} / \partial M^{\sim}\right) \times{ }_{G} E G$. We recover $M / \partial M$ as the homotopy orbits (i.e., reduced Borel construction) $\left(M^{\sim} / \partial M^{\sim}\right)_{h G}:=\left(M^{\sim} / \partial M^{\sim}\right) \wedge_{G} E G_{+}$. In its homotopy invariant formulation, the square is now given by the diagram of morphisms of $R(B G)$

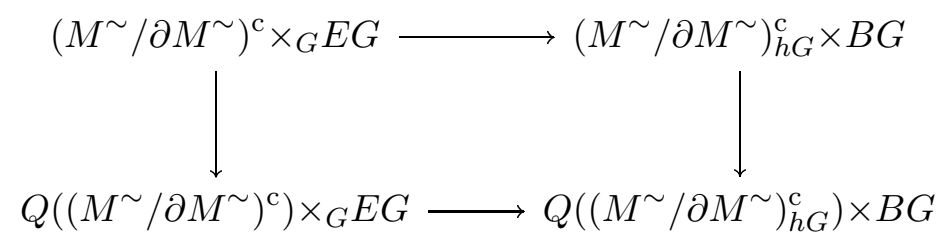

(here, for an object $Y \in R^{G}(*)$, the object $Y^{\mathrm{c}}$ denotes its cofibrant approximation).

Finally, we calculate the degree to which the square is cartesian. In what follows, set $N:=M^{\sim} / \partial M^{\sim}$, and note that $N$ is $(n-p-1)$-connected. The homotopy fiber of the left vertical map is the same thing as the homotopy fiber of the map $N \rightarrow Q N$. Denote this fiber by $F_{1}$. By Milgram's EHP-sequence [15, 1.11], there is a $(3 n-3 p-3)$-connected map $\Omega(N \wedge N)_{h \mathbb{Z} / 2} \rightarrow F_{1}$. On the other hand the homotopy fiber of the right vertical map is the same as the homotopy fiber of the map $N_{h G} \rightarrow Q\left(N_{h G}\right)$. If we denote this homotopy fiber by $F_{2}$, it again follows by Milgram's EHP-sequence that there is a $(3 n-3 p-3)$-connected map $\Omega\left(N_{h G} \wedge N_{h G}\right)_{h \mathbb{Z} / 2} \rightarrow F_{2}$. Moreover, the square

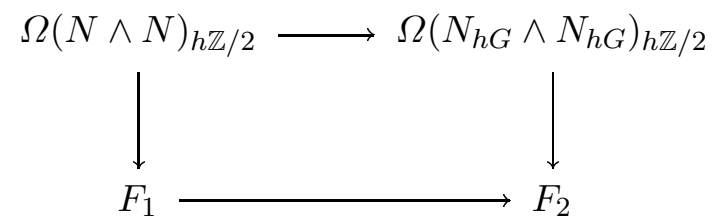

Algebraic 83 Geometric Topology, Volume 2 (2002) 
is commutative. The top map of the latter square is induced by the evident map $N \wedge N \rightarrow(N \wedge N)_{h G \times G}$. This last map is easily checked to be $(2 n-2 p+[p / 2])-$ connected. Assembling this information, it follows that the map $F_{1} \rightarrow F_{2}$ is $\min (3 n-3 p-4,2 n-2 p+[p / 2]-1)$-connected. By hypothesis, $3 p+4 \leq n$, so this connectivity is at least $n$. Consequently, the square (1) is $n$-cartesian, as claimed.

Proof of Theorem E The proof is similar to the proof of Theorem D (where here $P$ plays the role of $M$ ). Therefore, we will only sketch the argument and leave it to the reader to fill in the details.

As above, there is a diagram

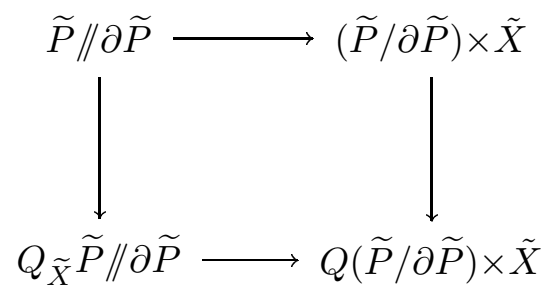

which one checks (by essentially the same argument) to be $(2 p)$-cartesian. The fiberwise stable normal invariant can be lifted to a fiberwise equivariant map $\widetilde{X} / \partial \widetilde{X} \rightarrow Q_{\widetilde{X}} \widetilde{P} / / \partial \widetilde{P}$. The rest of the argument follows as in the proof of Theorem B, substituting obstruction theory by equivariant obstruction theory, and using the fact that the equivariant homotopy dimension of $\widetilde{X} / \partial \widetilde{X}$ is $2 p$.

\section{Proof of Theorem A}

Our main tool will be the relative embedded thickening theorem of [10] (see also [11] for the absolute version). The statement of this result will require some preparation.

Let $(K, L)$ be a cofibration pair in Top. We assume for simplicity that $K$ and $L$ are cofibrant spaces which are homotopy finite. Write

$$
\operatorname{dim}(K, L) \leq k
$$

if there exists a factorization $L \rightarrow K^{\prime} \rightarrow K$ in which $K^{\prime}$ is obtained from $L$ by attaching cells of dimension $\leq k$ and the map $K^{\prime} \rightarrow K$ is a weak equivalence.

Let $X$ be an $n$-dimensional Poincaré space. 
By a relative embedded thickening of $(K, L)$ in $(X, \partial X)$ we mean a commutative diagram of cofibration pairs

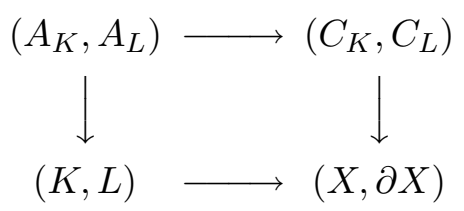

having the following properties.

- Each space appearing in the diagram is cofibrant and homotopy finite.

- Each of the diagrams of spaces

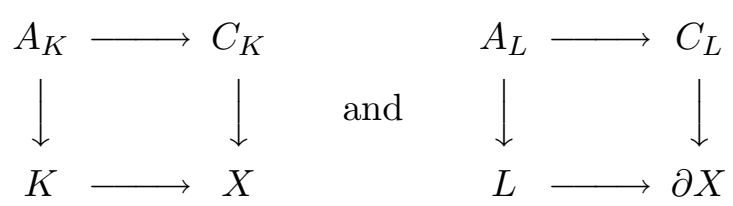

is cocartesian and the latter of these diagrams is a embedded thickening of $L$ in $\partial X$.

- The image of the fundamental class of $X$ with respect to the composite

$$
H_{n}(X, \partial X) \rightarrow H_{n}\left(\bar{X}, \partial X \cup_{C_{L}} C_{K}\right) \cong H_{n}\left(\bar{K}, L \cup_{A_{L}} A_{K}\right)
$$

gives $\left(\bar{K}, L \cup_{A_{L}} A_{K}\right)$ the structure of an $n$-dimensional Poincaré space (here, coefficients are given by pulling back the orientation bundle for $(X, \partial X))$. Similarly, $\left(\bar{C}_{K}, C_{L} \cup_{A_{L}} A_{K}\right)$ has the structure of a Poincaré space with fundamental class induced from $X$.

- The map $A_{K} \rightarrow K$ is $(n-k-1)$-connected.

The decomposition of $(X, \partial X)$ is depicted in figure 1 below.

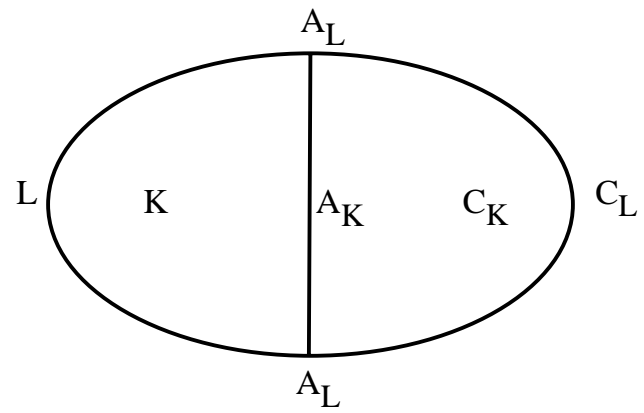

Figure 1

Now let $f:(K, L) \rightarrow(X, \partial X)$ be a map with $\operatorname{dim}(K, L) \leq k$ and suppose that the restriction $f_{\mid L}: L \rightarrow \partial X$ embedded thickens. The main theorem of [10] is 
Theorem 5.1 Assume $k \leq n-3$ and $f: K \rightarrow X$ is $(2 k-n+2)$-connected. Then there exists a relative embedded thickening of $f:(K, L) \rightarrow(X, \partial X)$ extending the given embedded thickening of $f_{\mid L}: L \rightarrow \partial X$.

Remark 5.2 The above is the Poincaré version of the relative embedded thickening theorem of Hodgson [8], with a loss of one dimension.

We now begin the proof of Theorem A. Assume $\left[\nu^{+}\right] \in\left[M^{+}, W\right]_{X}$ is trivial, where $W$ is the complement of an embedding of $M_{J}$ in $X_{I}$. We may also assume without loss in generality that $W \in R(X)$ is fibrant. A choice of fiberwise null-homotopy may be thought of as a family of maps $\nu_{t}: M_{t} \rightarrow W$ for $t \in[0,1 / 3]$ which commute with projection to $X$ such that $\nu=\nu_{1 / 3}$ and $\nu_{0}$ factors through $X_{0} \rightarrow W$.

This null-homotopy gives rise to a map of pairs

$$
\left(X_{0} \cup M_{[0,1 / 3]}, X_{0} \amalg M_{1 / 3}\right) \rightarrow(W, \partial W)
$$

in which $X_{0} \cup M_{[0,1 / 3]}$ is the mapping cylinder of the map $M_{1 / 3} \rightarrow X$. These circumstances are depicted in figure 2 .

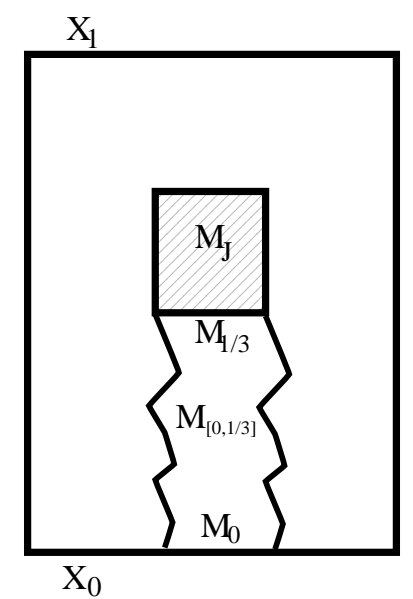

Figure 2

The restricted map of spaces

$$
X \amalg M \rightarrow \partial W
$$

is already embedded thickened (here, $\partial W=\partial X_{I} \amalg \partial M_{J}$ ). This embedded 
thickening is given by the cocartesian square

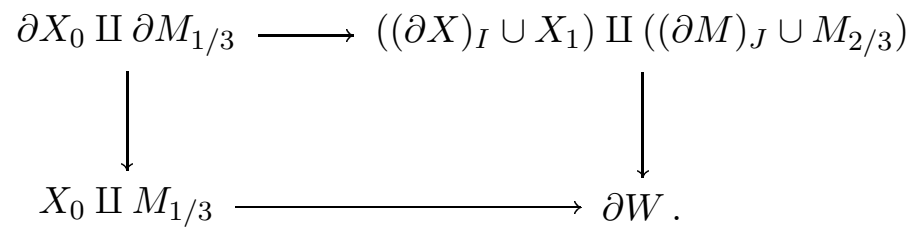

The map

$$
X_{0} \cup M_{[0,1 / 3]} \rightarrow W
$$

is $(n-p-1)$-connected (since it, followed by the map $W \rightarrow X_{I}$ is a weak equivalence, and the latter map is $(n-p)$-connected). Moreover, the pair $\left(X_{0} \cup\right.$ $\left.M_{[0,1 / 3]}, X \amalg M\right)$ has relative dimension $\leq p+1$.

Since $n-p-1 \geq 2(p+1)-(n+1)+2$ if and only if $2 n \geq 3 p+4$, by 5.1 there exists a relative embedded thickening of

$$
\left(X_{0} \cup M_{[0,1 / 3]}, X \amalg M\right) \rightarrow(W, \partial W)
$$

which extends the given embedded thickening of $X \amalg M \rightarrow \partial W$. Thus we have a diagram of pairs (cf. fig. 3)
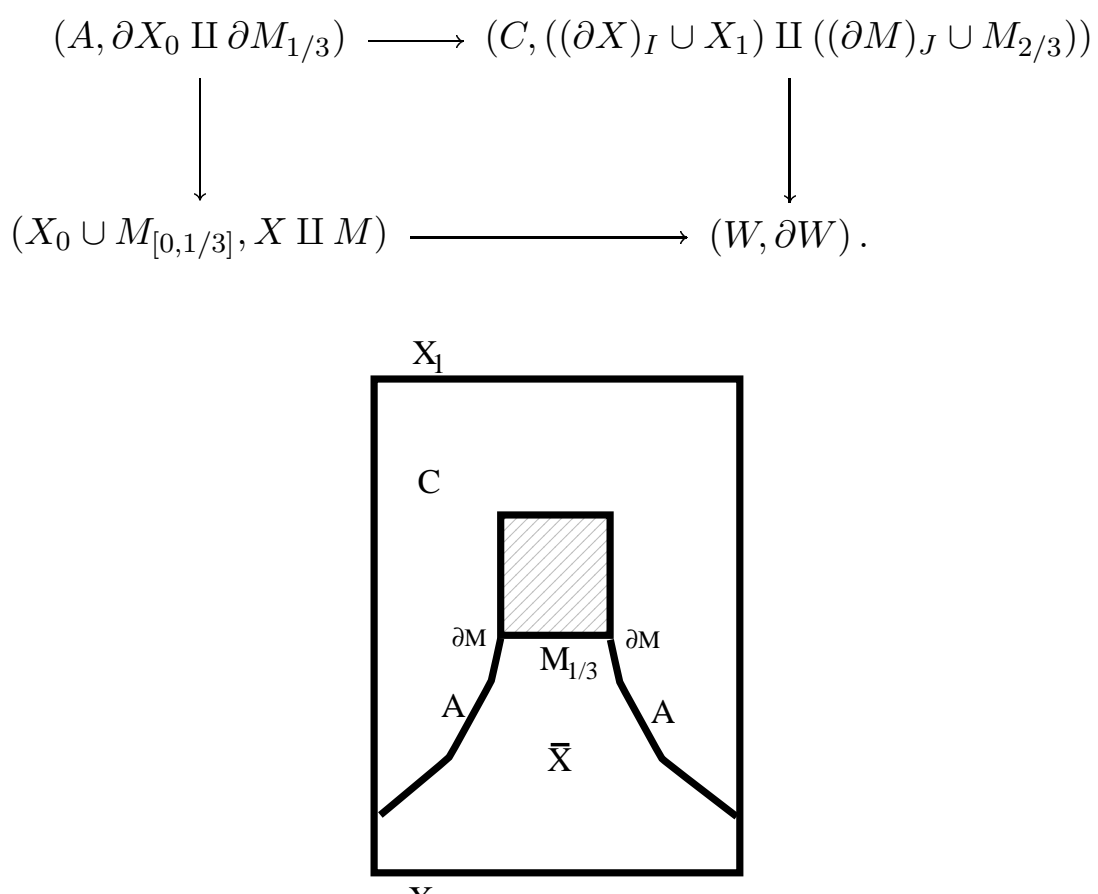

Figure 3

Algebraic 63 Geometric Topology, Volume 2 (2002) 
Consider the associated commutative diagram

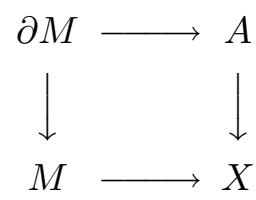

and note that there is an evident factorization of $\partial X \rightarrow X$ through the map $A \rightarrow X$.

To complete the proof of Theorem A, it suffices to show:

Claim The square (2) is an embedding of $M$ in $X$. It induces the given embedding of $M_{J}$ in $X_{I}$ after decompression.

To establish the claim, we first need to show that the square is cocartesian. According to the definitions $X_{0} \cup M_{[0,1 / 3]} \simeq X$ has an $n$-dimensional Poincaré boundary given by $X_{0} \cup_{\partial X_{0}}\left(M_{1 / 3} \cup_{\partial M_{1 / 3}} A\right)$. Application of Poincaré-Lefshetz duality gives an isomorphism

$$
H_{*}\left(\bar{X}, M \cup_{\partial M} A\right) \cong H^{n+1-*}\left(\bar{X}, X_{0}\right)=0 .
$$

in all degrees, for any bundle of coefficients on $X$. Moreover, the map $M \cup_{\partial M}$ $A \rightarrow \bar{X}$ induces an isomorphism on fundamental groups (since $A \rightarrow X$ and $\partial M \rightarrow M$ are 2-connected), so the square is cocartesian by application of Whitehead's theorem.

Secondly, a straightforward argument which we omit shows that the inclusion $X_{1} \subset C$ is a weak equivalence. Consequently, the composite $C \rightarrow W \rightarrow X$ is also a weak equivalence. Using this, we have a chain of weak equivalences

$$
\Sigma_{X} A \stackrel{\sim}{\rightarrow} X \cup_{A} C \stackrel{\sim}{\leftarrow} W
$$

which is compatible with projection to $X_{I}$ and is relative to $\partial X_{I}$. We infer that the decompression of (2) yields the embedding of $M_{J}$ in $M_{I}$ up to concordance. Compatibility of fundamental classes is a consequence of the remarks at the end of 2.3 and 2.4. This completes the proof of Theorem A.

\section{Theorem B: completion of the proof}

Given a fiberwise unstable normal invariant

$$
\alpha_{f} \in[X / / \partial X, M / / \partial M]_{X},
$$


we constructed in $\S 3$ an embedding of $M$ in $X$ by first associating an embedding of $M_{J}$ in $X_{I}$ and then applying Theorem A (using the observation that the compression obstruction of the latter embedding is trivial).

It remains to show that the collapse of this embedding coincides with $\alpha_{f}$. We will give the argument in the case when $\partial X=\emptyset$. The general case, which is straightforward, will be left to the reader.

Returning to the proof of Theorem A and in particular fig. 3 above, note that the collapse of the embedding of $M$ in $X$ is the fiberwise homotopy class of the map

$$
X_{0} \amalg\left(A \cup_{\partial M} M\right) \rightarrow \bar{X} \cup_{\partial M} M \quad\left(\stackrel{\sim}{\rightarrow} X \cup_{\partial M} M\right),
$$

whose restriction to $X_{0}$ is given by the inclusion $X_{0} \rightarrow \bar{X}$ and the restriction to $A \cup_{\partial M} M$ is given by the amalgamation of the map $A \rightarrow \bar{X}$ with the identity map of $M$.

Using fig. 3, we rewrite this as follows: consider the amalgamated union

$$
M^{\prime}:=(\partial M)_{J} \cup_{\partial M_{2 / 3}} M_{2 / 3} .
$$

and write $X^{\prime}:=A \cup_{\partial M_{1 / 3}} M^{\prime}$ (so $X^{\prime}$ is identified with $X$ up to weak equivalence). Then the fiberwise homotopy class of the composite

$$
X_{0} \amalg X^{\prime} \rightarrow \bar{X} \cup_{\partial M_{1 / 3}} M^{\prime} \stackrel{\sim}{\rightarrow} W
$$

represents the collapse of the embedding (recall that $W$ is $M / / \partial M$ made fibrant). Note there is an evident factorization $X_{0} \amalg X^{\prime} \rightarrow X_{0} \amalg C \rightarrow W$.

On the other hand, the composite

$$
X_{0} \amalg X_{1} \rightarrow X_{0} \amalg C \rightarrow W
$$

induces $\alpha_{f}$.

Consequently, the restrictions of the map $X \amalg C \rightarrow W$ to $X_{0} \amalg X^{\prime}$ and $X_{0} \amalg X_{1}$ induce respectively the collapse map of the embedding and $\alpha_{f}$.

But the maps $X_{1} \rightarrow C$ and $X^{\prime} \rightarrow C$ are weak homotopy equivalences. Consequently, the map $X_{0} \amalg C \rightarrow W$ induces both the collapse of the embedding of $M$ in $X$ and $\alpha_{f}$ on fiberwise homotopy. Thus $\alpha_{f}$ coincides with the collapse. This completes the proof of Theorem B. 


\section{Stability of the obstruction}

To prove 1.3 , we apply 2.3 to the homotopy set $\left[M^{+}, W\right]_{X}$. Since $M$ is homotopy equivalent to a complex of dimension $\leq p$, we infer that the object $M^{+} \in R(X)$ has dimension $\leq p$. On the other hand, the connectivity of $W \in R(X)$ is one less than the connectivity of the map $W \rightarrow X_{I}$, which in turn, is at least the connectivity of the map $\partial M_{J} \rightarrow M_{J}$ since the former is the cobase change of the latter. But $\operatorname{codim} M_{J} \geq n-p+1$, so $\partial M_{J} \rightarrow M_{J}$ is $(n-p)$-connected. Hence $W \in R(X)$ is an $(n-p-1)$-connected object.

Consequently, 2.3 implies that

$$
\left[M^{+}, W\right]_{X} \rightarrow\left[\Sigma_{X} M^{+}, \Sigma_{X} W\right]_{X}
$$

is an isomorphism whenever $p \leq 2(n-p-1)$, or equivalently, whenever $3 p+2 \leq$ $2 n$. Thus, the obstruction to compression is stable in the range of Theorem $\mathrm{A}$ (with two dimensions to spare).

\section{Proof of Theorem F}

In this section we show how Theorem A implies a partial improvement of the main result of [11]. Let $K$ be a cofibrant space which is homotopy equivalent to a connected $\mathrm{CW}$ complex of dimension $\leq k$. Let $X$ be a connected $n$ dimensional Poincaré space.

The main result of [11] is

Theorem 8.1 Assume that $f: K \rightarrow X$ is $(2 k-n+2)$-connected and $k \leq$ $n-3$. Then there exists an embedded thickening of $f$.

Now we have the statement of Theorem F, which is an improvement of 8.1 in the metastable range:

Theorem 8.2 Assume $f: K \rightarrow X$ is $(2 k-n+1)$-connected, $k \leq n-3$ and $3 k+4 \leq 2 n$. Then there exists an embedded thickening of $f$.

Proof By 8.1, there exists an embedded thickening of the composite $f_{I}: K \rightarrow$ $X=X_{0} \stackrel{\subset}{\hookrightarrow} X_{I}$. Let this be denoted

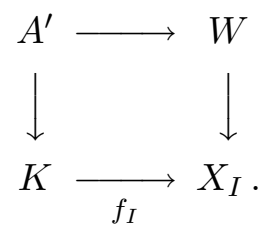

Algebraic $\mathcal{E} \mathcal{G}$ Geometric Topology, Volume 2 (2002) 
Without loss in generality, we may take $A^{\prime} \rightarrow K$ to be a fibration. By straightforward application of the Blakers-Massey theorem [5, p. 309], this square is $k$-cartesian. Let $P$ denote the homotopy pullback of the diagram given by deleting $A^{\prime}$. Then the evident map $A^{\prime} \rightarrow P$ is $k$-connected.

The maps id: $K \rightarrow K$ and $K \stackrel{f}{\rightarrow} X=X_{0} \subset W$ are compatible up to homotopy when followed by the given maps to $X_{I}$. Consequently, there is an induced map $K \rightarrow P$. As $A^{\prime} \rightarrow P$ is $k$-connected, we obtain a factorization $K \rightarrow A^{\prime} \rightarrow P$. Since $A^{\prime} \rightarrow K$ is a fibration, the homotopy lifting property plus the factorization yield a section $\zeta: K \rightarrow A^{\prime}$. By construction, the composite

$$
K^{+}=K \amalg X \stackrel{\zeta \amalg i d_{X}}{\longrightarrow} A^{\prime} \amalg X_{0} \rightarrow W
$$

is fiberwise null homotopic.

The map $\zeta: K \rightarrow A^{\prime}$ is $(n-k-1)$-connected. By 8.1 , it embedded thickens since $n-k-1 \geq 2 k-n+2$ is equivalent to $3 k+3 \leq 2 n$. Let

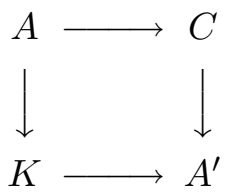

be such an embedded thickening. We claim that the composite $C \rightarrow A^{\prime} \rightarrow K$ is a weak equivalence. To see this, first note that $C \rightarrow K$ is 2-connected, since it is the composite of the $(n-k-1)$-connected map $C \rightarrow A^{\prime}$ with the $(n-k)$-connected map $A^{\prime} \rightarrow K$. Also, by Poincaré-Lefshetz duality, we infer that

$$
H_{*}(\bar{K}, C) \cong H^{n+1-*}(\bar{K}, K)=0
$$

in all degrees. Consequently, $C \rightarrow K$ is a weak equivalence by the Whitehead theorem.

Let $(M, \partial M)$ denote the pair $(\bar{K}, A)$. Then the argument of the last paragraph implies that $\left(M_{I}, \partial M_{I}\right)$ coincides with $\left(\bar{K}, A^{\prime}\right)$ up to homotopy. Furthermore, with respect to this homotopy equivalence, the inclusion $M_{0} \subset \partial M_{I}$ corresponds to $\zeta: K \rightarrow A^{\prime}$.

Assembling these data, we have an embedding of $M_{I}$ in $X_{I}$ whose obstruction $\left[\nu^{+}\right]$vanishes by (3). Applying Theorem A yields an embedded thickening of $f: K \rightarrow X$. 


\section{References}

[1] W Browder, Embedding 1-connected manifolds, Bull. Amer. Math. Soc. 72 (1966) 225-231

[2] W Browder, Embedding smooth manifolds, Proc. I.C.M. (1968) 712-719, moscow 1966

[3] J P Dax, Étude homotopique des espaces de plongements., Ann. Sci. École Norm. Sup. 5 (1972) 303-377

[4] TG Goodwillie, Multiple disjunction for spaces of homotopy equivalences, preprint, November 29, 1995

[5] T G Goodwillie, Calculus II: analytic functors, $K$-theory 5 (1992) 295-332

[6] A Hatcher, F Quinn, Bordism invariants of intersections of submanifolds, Trans. AMS 200 (1974) 327-344

[7] M Hirsch, Embeddings and compressions of smooth manifolds and polyhedra, Topology 4 (1966) 361-369

[8] J P E Hodgson, Obstructions to concordance for thickenings, Invent. Math. 5 (1970) 292-316

[9] I M James, Fibrewise homotopy theory, Handbook of Algebraic Topology, North Holland (1995) 169-194

[10] J R Klein, Poincaré embeddings and fiberwise homotopy theory, II, to appear in Quart. Jour. Math. Oxford

[11] J R Klein, Poincaré embeddings and fiberwise homotopy theory, Topology 38 (1999) 597-620

[12] J R Klein, Poincaré immersions, Forum Math. 11 (1999) 717-734

[13] J Levine, On differentiable imbeddings of simply-connected manifolds, Bull. AMS 69 (1963) 806-809

[14] N Levitt, Normal fibrations for complexes, Illinois J. Math. 14 (1970) 385-408

[15] R J Milgram, Unstable homotopy from the stable point of view, volume 368 of LNM, Springer (1974)

[16] D Quillen, Homotopical Algebra, volume 43 of LNM, Springer (1967)

[17] W Richter, A homotopy theoretic proof of Williams's Poincaré embedding theorem, Duke Math. J. 88 (1997) 435-447

[18] R Rigdon, B Williams, Embeddings and immersions of manifolds, Geometric applications of homotopy theory, Proc. Conf., Evanston, Ill. (1977), I, LNM 657 (1978) 423-454

[19] C T C Wall, Finiteness conditions for CW-complexes, Ann. of Math. 81 (1965) $55-69$

[20] C T C Wall, Poincaré complexes I, Ann. of Math. 86 (1967) 213-245

[21] C T C Wall, Surgery on Compact Manifolds, Academic Press (1970) 
[22] B Williams, Applications of unstable normal invariants I, Comp. Math. 38 (1979) 55-66

[23] B Williams, Hopf invariants, localizations, and embeddings of Poincaré complexes, Pacific J. Math. 84 (1979) 217-224

Department of Mathematics, Wayne State University Detroit, MI 48202, USA

Email: klein@math.wayne.edu

Received: 18 February 2002

Algebraic 6 Geometric Topology, Volume 2 (2002) 